\title{
Abstract strategies and coherence
}

\author{
Cameron Calk ${ }^{1}$, Eric Goubault ${ }^{1}$, and Philippe Malbos ${ }^{2}$ \\ 1 Laboratoire d'Informatique de l'École Polytechnique, École Polytechnique \\ 2 Institut Camille Jordan, Université Claude Bernard Lyon 1
}

\begin{abstract}
Normalisation strategies give a categorical interpretation of the notion of contracting homotopy via confluent and terminating rewriting. This approach relates standardisation to coherence results in the context of higher-dimensional rewriting systems. On the other hand, globular 2-Kleene algebras provide a formal setting for reasoning about coherence proofs in abstract rewriting systems. In this setting, we formalise the notion of normalisation strategy and we prove a formal coherence theorem for convergent abstract rewriting systems.
\end{abstract}

Keywords: Normalisation strategies · Kleene algebras · Formalisation - Coherence · Higher-dimensional rewriting.

\section{Introduction}

As pointed out in $[5,29]$ a central difficulty in formal mathematics is in balancing readability of specifications and proficient automated proof search. Capturing intuitions while remaining formally rigorous constitutes a first stumbling block, which ideally should result in a setting that provides correct, automated proofs which are readable and even illuminating. A powerful formalisation of abstract rewriting theory may be found in the theory of Kleene algebras. Algebraic abstraction allows for simple proofs in which deduction is replaced by calculation [29]. Proofs in this setting reconstruct intuitive proofs by diagrammatic reasoning, making Kleene algebras a formal setting well suited to capture abstract rewriting results. Modal Kleene algebras (MKAs) formalise abstract rewriting systems (ARS), abstractions of graphs of (1-dimensional) transitions, especially with respect to termination and normalisation properties [5, 29]. This setting does not suffice to formalise more subtle properties of normalisation strategies [24], such as standardisation properties, nor for dealing with inherently higher-dimensional transition systems. Indeed, these need a formalisation of equivalences between paths. This line of work started in [20,12], culminating in the introduction of a specific axiomatics on a 2-dimensional refinement of ARSs.

In this work, we are going one step further by giving a formalisation of a coherent extension of diagrammatic reasoning in the algebraic style of MKAs, inspired by coherent presentations in categorical algebra [23], or in algebra [10], 
and using a rewriting approach in the line of [27]. In a higher categorical structure, certain algebraic properties, e.g. associativity of composition, may only hold up to the existence of higher-dimensional morphisms. Given a collection of such higher morphisms, coherence is the requirement that the whole structure is contractible, i.e. all parallel morphisms are linked by higher morphisms. A coherence theorem states that, given a (generating) collection of such morphisms, coherence is satisfied. The objective is to obtain a minimal collection of generating higher morphisms. Graph-theoretical methods on string rewriting systems (SRS) were initiated by Squier in [27] to study coherence problems for monoids, a two dimensional word problem. The main point is to compute extensions of a SRS by homotopy generators which take the relations amongst the rewriting paths into account. That is, every pair of zig-zag sequences of rewriting paths with same source and same target can be paved by compositions of these generators. In Squier's approach, when the SRS is convergent, the homotopy generators are defined by the confluence diagrams of the critical branchings of the SRS. This rewriting method for coherence was applied to solve coherence problems in algebra [10,17,4], and for monoidal categories [14]. Thereby, the homotopy generators constitute the bottom part of a cofibrant replacement of the monoid presented by the SRS $[10,15]$. Squier's constructions were formulated in the categorical language of polygraphs in [16] for monoids and in [13] for higher categories.

In this work, we consider the case of ARS. The extension to the case of SRS will be done in a subsequent work because requires a formalisation of algebraic contexts and of the critical branching lemma, which constitutes a further development of the theory presented here. An ARS is represented by a quiver $\Phi$, aka a 1-polygraph, see Section 2. Parallel zig-zag sequences of rewriting paths are pairs of 1-cells in the free groupoid $\Phi^{\top}$ on $\Phi$ with same source and same target. Homotopical generators for the ARS consist of such pairs and form a cellular extension $X$ of $\Phi^{\top}$, see Section 2. The coherence theorem for $(\Phi, X)$ states that all parallel 1-cells in $\Phi^{\top}$ are equal modulo $X$. When $\Phi$ is convergent and $X$ is the set of confluence diagrams of (critical) branchings, Squier's method gives a proof of the coherence theorem for $\Phi$. It is exactly this proof that we formalise in this article.

This work uses the algebraic setting of a 2-dimensional (globular) version of MKAs, which model relation algebras and relations among relations, introduced in [3]. Interestingly enough, these 2-dimensional MKAs are close to Concurrent Kleene Algebras (CKAs), which introduce an extra algebraic operation modelling parallel composition, hence equivalences between (1-dimensional) paths.

Structure of the article, and main results. This article is about formalising normalisation strategies and coherence properties in view of automating proofs. In Section 2, we present the categorical formulation of relations among relations in terms of cellular tilings, and based on Squier's coherence result. We then recap the MKA approach for ARS in Section 3. Coherent rewriting in globular modal 2-Kleene algebra, which we introduced in [3], is recalled in Sections 4 and 5. Sections 6 and 7 form the core of our new results, where we first model normal- 
isation strategies in 2-MKAs, and prove abstract coherence properties therein. Our first result, Theorem 1, gives a formalisation of a coherent normalising Newman's lemma. We thereby deduce our main result, Theorem 2 , which formalises a proof of contractibility via normalisation strategies.

\section{Squier's theorem for ARS}

We consider an ARS as a quiver, i.e. a directed graph with parallel and looping transitions, which we call a 1-polygraph from the terminology of higherdimensional rewriting $[2,28]$. Denote by $\Phi:=\left(\Phi_{0}, \Phi_{1}\right)$ a 1 -polygraph with source and target maps $s_{0}, t_{0}: \Phi_{1} \rightarrow \Phi_{0}$. We model the reflexive, transitive closure of $\Phi$ by the free 1-category $\Phi^{*}$ generated by $\Phi$, the underlying graph of which consists of the directed paths in $\Phi$. Similarly, the symmetric, reflexive, transitive closure of $\Phi$ is modelled by the free 1-groupoid $\Phi^{\top}$ generated by $\Phi$, its underlying graph consisting of undirected paths. In both cases the source and target maps are obtained by naturally extending those of $\Phi$. The vertices (resp. edges) of such structures will henceforth be referred to as 0-cells (resp. 1-cells), and the set of $i$-cells of $\Phi^{*}\left(\operatorname{resp} . \Phi^{\top}\right)$ will be denoted by $\Phi_{i}^{*}\left(\operatorname{resp} . \Phi_{i}^{\top}\right)$. The 0 -composition of 1-cells $x, y$ is defined when $t_{0}(x)=s_{0}(y)$ and is denoted by $x \star_{0} y$. The identity 1-cell on $a \in \Phi_{0}$ is denoted by $1_{a}$ and the inverse of a 1-cell $x$ is denoted by $x^{-}$. Two 1-cells are parallel when they have the same 0 -source and 0-target. Directed paths correspond to compositions $x_{1} \star_{0} \cdots \star_{0} x_{k}$, with $x_{i} \in \Phi_{1}$. Similarly, undirected paths correspond to finite compositions of elements of $\Phi_{1}$ and their formal inverses, quotiented by the relations $x \star_{0} x^{-} \sim 1_{s_{0}(x)}$, for $x \in \Phi_{1}$.

A cellular extension $X$ of $\Phi^{\top}$ is a quiver on the edges of $\Phi^{\top}$, i.e. a pair $\left(\Phi_{1}^{\top}, X\right)$ with source (resp. target) map $s_{1}$ (resp. $t_{1}$ ), such that the globular relations $t_{0} \circ s_{1}=t_{0} \circ t_{1}$ and $s_{0} \circ s_{1}=s_{0} \circ t_{1}$ are satisfied. The elements of $X$ are called generating 2-cells and may be thought of as (directed) tiles filling the space between parallel 1-cells. The pair $(\Phi, X)$ is called a $(2,0)$-polygraph.

Recall that the 2-cells in a 2-category may be composed in two different ways. The 0-composition of $\gamma: x \Rightarrow y$ and $\delta: x^{\prime} \Rightarrow y^{\prime}$, where $x, y: a \rightarrow b$ and $x^{\prime}, y^{\prime}: b \rightarrow c$ are pairs of parallel 1-cells, is a 2-cell $\gamma \star_{0} \delta: x \star_{0} x^{\prime} \Rightarrow y \star_{0} y^{\prime}$. The 1-composition of 2-cells $\alpha: x \Rightarrow y$ and $\beta: y \Rightarrow z$, where $x, y, z$ are parallel 1 -cells, is a 2-cell $\alpha \star_{1} \beta: x \Rightarrow z$. A 2-groupoid is a 2-category in which all 1and 2-cells are invertible for 0 - and 1-composition, respectively. Given a $(2,0)$ polygraph $(\Phi, X)$, we consider the free 2-groupoid generated by $(\Phi, X)$, denoted by $X^{\top}$, which has $\Phi^{\top}$ as its underlying 1-groupoid and containing all finite 0 and 1-compositions of the generating 2-cells in $X$ and their inverses, as well as 0-compositions with 1-cells of $\Phi^{\top}$.

The confluence properties of an ARS $\Phi$ can be stated with respect to a cellular extension $X$ of $\Phi^{\top}$. This approach first appeared in [20] under the terminology of commuting diagrams. A local branching $(x, y)$ of $\Phi$ is $X$-confluent if there exist 1 -cells $x^{\prime}, y^{\prime}$ in $\Phi_{1}^{*}$, and a 2-cell $\alpha$ in the free 2-groupoid $X^{\top}$ as in the adjacent diagram. The ARS $\Phi$ is locally $X$-confluent when

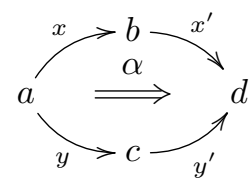


every local branching of $\Phi$ is $X$-confluent. We say that parallel 1-cells $f$ and $g$ of $\Phi^{\top}$ are $X$-congruent if there exists a 2-cell $\alpha: f \Rightarrow g$ in $X^{\top}$, and that $(\Phi, X)$ is acyclic if all parallel 1-cells of $\Phi^{\top}$ are $X$-congruent. The ARS $\Phi$ terminates if it contains no infinite directed paths.

Let us recall the proof that a terminating, locally $X$-confluent $(2,0)$-polygraph $(\Phi, X)$ is acyclic. Firstly note that if an ARS $\Phi$ is locally $X$-confluent then it is locally confluent so, under the hypothesis of termination, is confluent by Newman's lemma. In this case, from every 0-cell a, a normal form, i.e. a 0-cell irreducible by $\Phi$, may be reached in a finite number of steps. Since $\Phi$ is confluent, the normal form of $a$ is unique; we denote it by $\hat{a}$.

By local $X$-confluence and termination, we may therefore choose, for every 0 -cell $a$ of $\Phi$, a 1-cell $\sigma_{a}: a \rightarrow \hat{a}$ in $\Phi_{1}^{*}$. A normalisation strategy $\sigma$ is a function $\Phi_{0} \rightarrow \Phi_{1}^{\top}$ which assigns such a $\sigma_{a}$ to every 0 -cell $a$, under the condition that $\sigma_{b}=1_{b}$ for any normal form $b$. Just as normal forms provide a representative 0 -cell for connected components in $\Phi^{\top}$, a normalisation strategy is the given of a representative 1-cell in $\Phi^{\top}$ among parallel reductions to normal forms.

Now that we are equipped with a normalisation strategy $\sigma$, we prove by Noetherian induction on the distance from a normal form that for any branching $(x, y)$ of $\Phi^{*}$, there exists a 2-cell $\alpha$ as in the adjacent diagram. When $s_{0}(x)=s_{0}(y)$ is a normal form, we

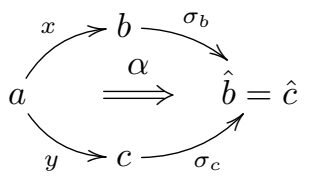
can simply use identity 1 - and 2-cells to obtain the desired diagram. For the induction step, we observe that we can write $x$ as $x=x_{1} \star_{0} x_{r}$, where $x_{1}$ is a 1-cell of $\Phi$ and $x_{r}$ is one step closer to a normal form, and similarly for $y$. By the hypothesis of local confluence and the Noetherian induction hypothesis, we obtain the result by composing the 2-cells in the diagram on the left below:
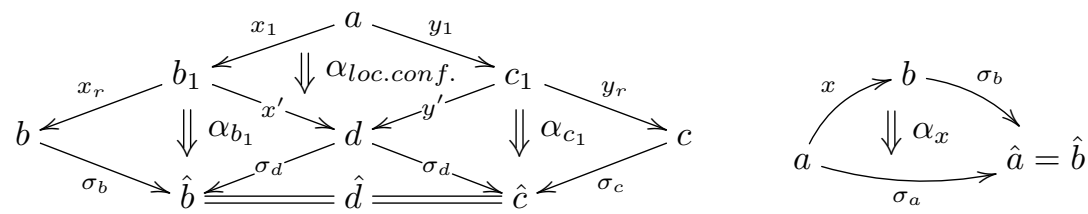

Let $x: a \rightarrow b$ be a 1 -cell of $\Phi^{*}$, consider the branching $\left(x \star_{0} \sigma_{b}, \sigma_{a}\right)$ of $\Phi^{*}$. Since we cannot reduce any further than normal forms, by the above result, as well as a rotation of the 2-cell by properties of 2-groupoids, we obtain a 2-cell $\alpha_{x}$ as pictured above on the right. A similar 2-cell for all inverses of 1-cells may be found, again using properties of 2-groupoids which we will not develop here. Note that every 1-cell $f: a \rightarrow b$ of $\Phi^{\top}$ can be factorised as $f=x_{1} \star_{0} y_{1}^{-} \star_{0} \cdots \star_{0} x_{p} \star_{0} y_{p}^{-}$, where the $x_{i}$ and $y_{i}$ are 1-cells of $\Phi^{*}$. Denote by $\alpha_{f}$ the composite 2-cell of $X^{\top}$ :

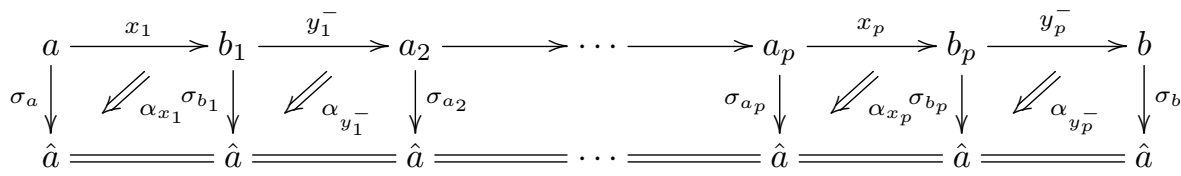

Compiling all of the above, we obtain the coherence theorem for ARS: 
Theorem A. Let $\Phi$ be a terminating ARS and $X$ be a cellular extension of $\Phi^{\top}$. If $\Phi$ is locally $X$-confluent, then for every 1-cell $f: a \rightarrow b$ of $\Phi^{\top}$, there exists a 2 -cell $\alpha_{f}: f \star_{0} \sigma_{b} \Rightarrow \sigma_{a}$ in the free 2-groupoid generated by $(\Phi, X)$.

Squier's theorem [27] is deduced from the above result. Indeed, we prove that for all parallel 1-cells $f, g: a \rightarrow b$ of $\Phi^{\top}$, the composite 2-cell

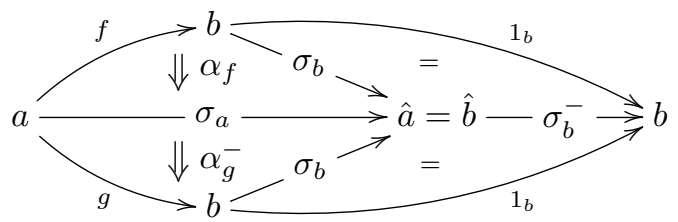

in $X^{\top}$ has source $f$ and target $g$. This proves that the pair $(\Phi, X)$ is acyclic.

Theorem B. Let $\Phi$ be a terminating ARS and $X$ be a cellular extension of $\Phi^{\top}$. If $\Phi$ is locally $X$-confluent, then $(\Phi, X)$ is acyclic.

This is Squier's formulation of the coherence theorem for ARSs, and is an immediate consequence of Theorem A, relying solely on the definitions of acyclicity and of 2-groupoids.

\section{Modal 1-Kleene algebras}

In order to fix notation, we recall the definitions of Boolean modal Kleene algebras from [6,5] and of converse from [1]. We adapt one of the converse axioms in order to establish a natural relationship between domain and conversion akin to that of inverse semigroups, see e.g. [22].

Semirings. A semiring is a structure $(S,+, 0, \cdot, 1)$ such that $(S,+, 0)$ is a commutative monoid, $(S, \cdot, 1)$ is a monoid whose multiplication . (often denoted by juxtaposition) distributes on the left and the right over the addition + , and 0 is a left and right annihilator for $\cdot$. A dioid is a semiring in which addition is idempotent. In this case, the relation defined by $x \leq y \Leftrightarrow x+y=y$, for all $x, y \in S$, is a partial order on $S$, with respect to which addition and multiplication are monotone, and for which 0 is the minimum.

(Boolean) domain semirings. A domain semiring is a dioid $S$ equipped with a domain operation $d: S \rightarrow S$ satisfying the following five axioms for all $x, y \in S$ :

$x \leq d(x) x, \quad d(x y)=d(x d(y)), \quad d(x) \leq 1, \quad d(0)=0, \quad d(x+y)=d(x)+d(y)$.

The set $S_{d}$ of fixpoints of $d$ forms a distributive lattice with + as join and · as meet, bounded by 0 and 1 . We write $p, q, r, \ldots$ for elements of $S_{d}$ and refer to $S_{d}$ as the domain algebra of $S$. A Boolean domain semiring is a dioid $S$ equipped with an antidomain operation ad $: S \rightarrow S$ satisfying the following three axioms:

$$
a d(x) x=0, \quad a d(x y) \leq a d\left(x a d^{2}(y)\right), \quad a d^{2}(x)+a d(x)=1,
$$


for all $x, y \in S$. Setting $d=a d^{2}$, we recover a domain semiring. In the presence of an antidomain, $S_{d}=a d(S)$ and $a d$ acts as Boolean complementation on $S_{d}$. We denote the restriction of $a d$ to $S_{d}$ by $\neg$.

Modal semirings. We denote by $S^{o p}$ the opposite of a dioid $S$, in which the order of multiplication has been reversed. A codomain (resp. Boolean codomain) semiring is a dioid equipped with a map $r: S \rightarrow S$ (resp. ar $: S \rightarrow S$ ) such that $\left(S^{o p}, r\right)$ (resp. $\left.\left(S^{o p}, a r\right)\right)$ is a domain (resp. Boolean domain) semiring. A modal semiring is a dioid $S$ which is both a domain and codomain semiring, and satisfies for every $x \in S, d(r(x))=r(x)$ and $r(d(x))=d(x)$.

Modal Kleene algebras. A Kleene algebra is a dioid $K$ equipped with an operation $(-)^{*}: K \rightarrow K$ called the Kleene star, satisfying the following axioms:

i) $1+x x^{*} \leq x^{*}$ and $1+x^{*} x \leq x^{*}$ (unfold axioms),

ii) $z+x y \leq y \Rightarrow x^{*} z \leq y$ and $z+y x \leq y \Rightarrow z x^{*} \leq y$ (induction axioms),

for all $x, y, z \in K$. The Kleene plus is defined by $x^{+}=x x^{*}$. (Anti-)domain and (anti-)codomain operations extend to Kleene algebras without additional axioms. We thus define a (Boolean) modal Kleene algebra, or (Boolean) MKA for short, as a Kleene algebra that is also a (Boolean) modal semiring.

Converse. A Kleene algebra with converse [1] is a Kleene algebra $K$ equipped with an involution $\overline{(-)}: K \rightarrow K$ that satisfies, for all $x, y \in K$,

$$
\overline{(x+y)}=\bar{x}+\bar{y}, \quad \overline{(x \cdot y)}=\bar{y} \cdot \bar{x}, \quad \overline{\left(x^{*}\right)}=(\bar{x})^{*}, \quad \overline{(\bar{x})}=x,
$$

and the inequality $x \leq x \bar{x} x$. In this work, we alter the final axiom in order to relate conversion to the domain operation. We consider an involution $\overline{(-)}: K \rightarrow$ $K$ satisfying axioms (1) and

$$
x \bar{x} \geq d(x),
$$

a similar axiom to that found in inverse semigroups [22]. We observe that such a converse operation exchanges domain and codomain, i.e. $d(\bar{x})=r(x)$ and $r(\bar{x})=d(x)$, and that for $p \in K_{d}, \bar{p}=p$. A (Boolean) MKA with converse is a (Boolean) MKA equipped with such a converse operation.

Modalities in dimension one. Let $K$ be a MKA. For $x \in K$ and $p \in K_{d}$, we define modal forward and backward diamond operators:

$$
|x\rangle p=d(x p), \quad\langle x| p=r(p x) .
$$

When a statement holds for both forward and backward diamonds, we will write $\langle x\rangle$. Note that by monotonicity of domain, the assignment $x \mapsto\langle x\rangle$ is monotone for the point-wise order on operators. When $K$ is a Boolean MKA, we additionally define modal box operators:

$$
\mid x] p=\neg|x\rangle(\neg p), \quad[x \mid p=\neg\langle x|(\neg p) .
$$


These are modal operators in the sense of Boolean algebras with operators [21]. For $K$ with converse, we have $|\bar{x}\rangle=\langle x|$ and $\langle\bar{x}|=| x\rangle$, and similarly for boxes. Boxes and diamonds form a Galois connection, i.e.

$$
|x\rangle p \leq q \Leftrightarrow p \leq[x \mid q \quad \text { and } \quad\langle x|p \leq q \Leftrightarrow p \leq| x] q .
$$

We have $|x y\rangle=|x\rangle \circ|y\rangle,\langle x y|=\langle y|\circ\langle x|| x y]=| x,] \circ| y]$ and $[x y \mid=[y \mid \circ[x \mid$ for all $x, y \in K$; in what follows we will denote functional composition of modal operators simply by juxtaposition. Finally, star unfold and induction axioms lift to modalities:

$$
\begin{gathered}
|1\rangle+|x\rangle\left|x^{*}\right\rangle=\left|x^{*}\right\rangle, \quad|1\rangle+|x\rangle\left|x^{*}\right\rangle=\left|x^{*}\right\rangle, \\
|y\rangle+|x\rangle|z\rangle \leq|z\rangle \Rightarrow\left|x^{*}\right\rangle|y\rangle \leq|z\rangle,
\end{gathered}
$$

where the addition is the point-wise lifting of that in $K_{d}$.

Rewriting and modal Kleene algebras. We recall from [5] formalised properties of ARS expressed in MKA. An element $x \in K$ terminates, or is Noetherian, provided that for all $p \in K_{d}$ the implication $p \leq|x\rangle p \Rightarrow p=0$ holds. The set of Noetherian elements of $K$ is denoted by $\mathcal{N}(K)$. The Galois connections (4) yield the following equivalent characterisation of termination:

$$
\left.\forall p \in K_{d}, \quad \mid x\right] p \leq p \Rightarrow p=1 .
$$

The exhaustion of an element $x \in K$, denoted by $\operatorname{exh}(x)$, is defined by

$$
\operatorname{exh}(x):=x^{*} \cdot \neg d(x) .
$$

The normal forms element of $x \in K$, denoted by $\mathrm{nf}_{x}$, is defined by

$$
\mathrm{nf}_{x}:=r(\operatorname{exh}(x)) \in K_{d} .
$$

Confluence properties are captured in MKA by semi-commutation. Given $x, y \in$ $K$, we say that the ordered pair $(x, y)$ semi-commutes locally if $x y \leq y^{*} x^{*}$, semicommutes if $x^{*} y^{*} \leq y^{*} x^{*}$, and has the Church-Rosser property if $(x+y)^{*} \leq$ $y^{*} x^{*}$. An element $x \in K$ is (locally) confluent (resp. Church-Rosser) if the pair $(\bar{x}, x)$ semi-commutes (resp. has the Church-Rosser property). We say that $x$ is convergent if it is both terminating and confluent. These properties are related to exhaustion as follows:

Lemma 1 ([5]). Let $K$ be a Boolean modal Kleene algebra and $x \in K$. If $x$ terminates, then $d(\operatorname{exh}(x))=1$. If $x$ is confluent, then exh $(x)$ is deterministic, i.e. $\langle\operatorname{exh}(x)|| \operatorname{exh}(x)\rangle \leq\langle 1\rangle$.

\section{Globular 2-Kleene algebras}

In [3], the notion of $p$-Boolean globular $n$-Kleene algebra was introduced as a higher-dimensional extension of MKAs. Here we briefly recall the case of $p=0$ and $n=2$, and append the notion of converse. 
A modal 2-Kleene algebra is a structure $\left(K,+, 0, \odot_{i}, 1_{i}, d_{i}, r_{i},(-)^{*_{i}}\right)_{i=0,1}$, such that for each $i \in\{0,1\}, K$ is a MKA with respect to $i$-operations, and in which the following additional axioms hold:

i) (2-dioid axioms) The lax interchange law: for all $A, A^{\prime}, B, B^{\prime} \in K$,

$$
\left(A \odot_{1} A^{\prime}\right) \odot_{0}\left(B \odot_{1} B^{\prime}\right) \leq\left(A \odot_{0} B\right) \odot_{1}\left(A^{\prime} \odot_{0} B^{\prime}\right),
$$

and the 1 -unit is an idempotent for 0 -multiplication, i.e. $1_{1} \odot_{0} 1_{1}=1_{1}$. Note that these correspond to the standard concurrent semiring axioms [18], except that the equality $1_{0}=1_{1}$ is normally assumed in this case.

ii) (Domain 2-semiring axioms) The (co-)domain operations satisfy absorption axioms $d_{1} \circ d_{0}=d_{0}$ and $r_{1} \circ r_{0}=r_{0}$. The set $K_{d_{i}}$ is called the $i$-dimensional domain algebra, and is denoted by $K_{i}$.

iii) (Kleene star axioms) The 1-star $(-)^{*_{1}}$ is a lax morphism with respect to 0 -multiplication of 1-dimensional elements on the right (resp. left), i.e. for all $A \in K$ and $\phi \in K_{1}$,

$$
\phi \odot_{0} A^{*_{1}} \leq\left(\phi \odot_{0} A\right)^{*_{1}}, \quad\left(\text { resp. } A^{*_{1}} \odot_{0} \phi \leq\left(A \odot_{0} \phi\right)^{*_{1}}\right) .
$$

For more details, see [3]. In order to distinguish elements of distinct dimensions, we denote elements of $K_{0}$ by $p, q, r, \ldots$, elements of $K_{1}$ by $\phi, \psi, \xi, \ldots$, and general elements of $K$ by $A, B, C, \ldots$.

As additional conditions, we may ask that a modal 2-Kleene algebra be globular, Boolean or equipped with converses. These notions are recalled below.

Globular axioms. A modal 2-Kleene algebra $K$ is globular if the following globular relations hold for all $A, B \in K$ :

$$
\begin{aligned}
& d_{0} \circ d_{1}=d_{0} \text { and } d_{0} \circ r_{1}=d_{0}, \quad d_{1}\left(A \odot_{0} B\right)=d_{1}(A) \odot_{0} d_{1}(B), \\
& r_{0} \circ d_{1}=r_{0} \text {, and } r_{0} \circ r_{1}=r_{0}, \quad r_{1}\left(A \odot_{0} B\right)=r_{1}(A) \odot_{0} r_{1}(B) \text {. }
\end{aligned}
$$

As a consequence of the rightmost axioms, $K_{1}$ is a MKA with respect to 0-operations. An element $A$ of $K$ will be represented graphically by the adjacent diagram with respect to its 0 - and 1-domains and codomains.

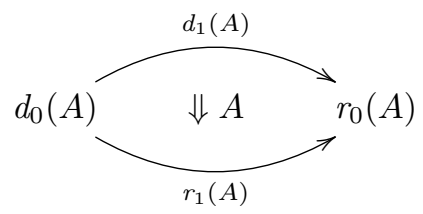

Boolean axioms. A modal 2-Kleene algebra is Boolean if it is augmented with maps $a d_{0}: K \rightarrow K$ and $a r_{0}: K \rightarrow K$, such that $\left(K,+, 0, \odot_{0}, 1_{0}, a d_{0}, a r_{0}\right)$ is a Boolean MKA, i.e. $a d_{0}$ (resp. $\left.a r_{0}\right)$ satisfies the antidomain (resp. anticodomain) axioms and $d_{0}=a d_{0}^{2}\left(\right.$ resp. $\left.r_{0}=a r_{0}^{2}\right)$. The domain algebra $K_{0}$ is thus a Boolean algebra whose complementation, denoted by $\neg$, is given by the restriction of $a d_{0}$ (and $a r_{0}$ ) to $K_{0}$. 
Converses. We will consider modal 2-Kleene algebras with 0-converses, i.e. equipped with an operation $\overline{(-)}: K_{1} \rightarrow K_{1}$ such that $\left(K_{1},+, 0, \odot_{0}, 1_{0},(-)^{*}, \overline{(-)}\right)$ is a MKA with converse. For a more general notion of converse in higherdimensional Kleene algebra, we refer the reader to [3].

Modalities in 2-semirings. Recall from [3], that the $i$-diamond operators of a modal 2-Kleene algebra $K$ are defined via the (co-)domain operators in each dimension. For $i \in\{0,1\}, A \in K$ and $\phi \in K_{i}$,

$$
|A\rangle_{i}(\phi)=d_{i}\left(A \odot_{i} \phi\right), \quad \text { and } \quad\left\langle\left. A\right|_{i}(\phi)=r_{i}\left(\phi \odot_{i} A\right) .\right.
$$

These modal operators have all of the properties recalled in Section 3 with respect to $i$-operations and elements of $K_{i}$. Since we are considering Boolean modal 2-Kleene algebras we may additionally define 0-boxes.

Polygraphic model. Let $(\Phi, X)$ be a $(2,0)$-polygraph. We define $K(\Phi, X)$, the full 2-path algebra over $(\Phi, X)$ as follows. Let $X_{2}^{\top}$ denote the set of 2-cells in $X^{\top}$. The carrier set of $K(\Phi, X)$ is the power set $\mathcal{P}\left(X_{2}^{\top}\right)$, whose elements, denoted by $A, B, C \ldots$ are sets of 2-cells, which in turn are denoted by $\alpha, \beta, \gamma \ldots$ Recall that for each 1-cell $x$ of $X^{\top}$, there exists a unique 2-cell $1_{x}$, its identity 2-cell, and similarly, for each 0 -cell $a$ there exists a unique 2-cell $1_{1_{a}}$, the identity 2 -cell on its identity 1 -cell. For $i \in\{0,1\}$, the $i$-composition, $i$-source and $i$-target maps are thereby defined for cells of any dimension.

For $i \in\{0,1\}$, the multiplication $\odot_{i}$ on $K(\Phi, X)$ is the lifting of the composition operations of $X^{\top}$ to the power-set, i.e. for any $A, B \in K(\Phi, X)$,

$$
A \odot_{i} B:=\left\{\alpha \star_{i} \beta \mid \alpha \in A \wedge \beta \in B \wedge t_{i}(\alpha)=s_{i}(\beta)\right\} .
$$

The units are the sets $\mathbb{1}_{0}=\left\{1_{1_{a}} \mid a \in \Phi_{0}\right\}$, and $\mathbb{1}_{1}=\left\{1_{x} \mid x \in \Phi_{1}^{\top}\right\}$. The addition in $K(\Phi, X)$ is given by set union; the ordering is therefore given by set inclusion. The domain and codomain maps are defined by

$$
\begin{aligned}
& d_{0}(A):=\left\{1_{1_{s_{0}(\alpha)}} \mid \alpha \in A\right\}, \quad r_{0}(A):=\left\{1_{1_{t_{0}(\alpha)}} \mid \alpha \in A\right\}, \\
& d_{1}(A):=\left\{1_{s_{1}(\alpha)} \mid \alpha \in A\right\}, \quad \text { and } \quad r_{1}(A):=\left\{1_{t_{1}(\alpha)} \mid \alpha \in A\right\} \text {, }
\end{aligned}
$$

and are thus given by lifting the source and target maps of $X^{\top}$ to the power set. The $i$-antidomain and $i$-anticodomain maps are then given by complementation with respect to the set of $i$-cells. The $i$-star is given by $A^{*_{i}}=\bigcup_{k \in \mathbb{N}} A^{k_{i}}$, where in the above, $A^{0_{i}}:=\mathbb{1}_{i}$ and $A^{k_{i}}:=A \odot_{i} A^{(k-1)_{i}}$. For $\psi \in K(\Phi, X)_{1}$, the converse is given by $\bar{\psi}:=\left\{1_{x^{-}} \mid 1_{x} \in \psi\right\}$.

Proposition 1 ([3]). Let $(\Phi, X)$ be a $(2,0)$-polygraph. Then, $K(\Phi, X)$ is a globular Boolean modal 2-Kleene algebra. 


\section{Coherent rewriting and modal 2-Kleene algebras}

We fix $K$ a globular 2-Kleene algebra. Given $A \in K$ and $\phi, \phi^{\prime} \in K_{1},|A\rangle_{1}(\phi) \geq \phi^{\prime}$ is equivalent to $d_{1}\left(A \odot_{1} \phi\right) \geq \phi^{\prime}$ by definition. In terms of quantification over collections of cells, this means that for every $u$ in $\phi^{\prime}$, there exist $v$ in $\phi$ and $\alpha$ in $A$ such that the 1-source (resp. 1-target) of $\alpha$ is $u$ (resp. $v$ ). This observation motivates the following definitions from [3]. For $\phi, \psi$ in $K_{1}$, an element $A$ in $K$ is a local confluence filler for $(\phi, \psi)$ if $|A\rangle_{1}\left(\psi^{*_{0}} \odot_{0} \phi^{*_{0}}\right) \geq \phi \odot_{0} \psi$, is a confluence filler for $(\phi, \psi)$ if $|A\rangle_{1}\left(\psi^{*_{0}} \odot_{0} \phi^{*_{0}}\right) \geq \phi^{*_{0}} \odot_{0} \psi^{*_{0}}$, and is a Church-Rosser filler for $(\phi, \psi)$ if $|A\rangle_{1}\left(\psi^{*_{0}} \odot_{0} \phi^{* 0}\right) \geq(\psi+\phi)^{*_{0}}$.

The right (resp. left) whiskering of an element $A \in K$ by $\phi \in K_{1}$ is the element $A \odot_{0} \phi$ (resp. $\phi \odot_{0} A$ ). Recall from [3] that whiskering commutes with 1-diamonds, that is, for all $A \in K$ and $\phi, \psi, \phi^{\prime}, \psi^{\prime}, \gamma \in K_{1}$ such that $\phi^{\prime} \leq \phi$, $\psi^{\prime} \leq \psi$, and $d_{1}(A) \leq \gamma$, we have:

$$
\phi^{\prime} \odot_{0}|A\rangle_{1}(\gamma) \odot_{0} \psi^{\prime}=\left|\phi^{\prime} \odot_{0} A \odot_{0} \psi^{\prime}\right\rangle_{1}\left(\phi \odot_{0} \gamma \odot_{0} \psi\right) .
$$

Fix a (local) confluence filler $A$ of a pair $(\phi, \psi)$ of elements in $K_{1}$. The total whiskering of $A$, denoted by $\hat{A}$, is the following element of $K$ :

$$
\hat{A}:=(\phi+\psi)^{*_{0}} \odot_{0} A \odot_{0}(\phi+\psi)^{*_{0}} .
$$

The 1-star of $\hat{A}$ is called the completion of $A$. Note that this element absorbs whiskers, that is, for every $\xi \leq(\phi+\psi)^{*_{0}}$,

$$
\xi \odot_{0} \hat{A}^{*_{1}} \leq \hat{A}^{*_{1}} \quad \text { and } \quad \hat{A}^{*_{1}} \odot_{0} \xi \leq \hat{A}^{*_{1}} .
$$

\section{Formalisation of normalisation strategies}

In this section, we formalise the notion of normalisation strategy, introduced in [15]. We first define notions of section, skeleton and strategy in one-dimensional Kleene algebras and show properties thereof. In what follows, we consider a Boolean MKA $K$ with converse and an element $x \in K$.

i) The equivalence generated by $x$ is the element $x^{\top}:=(x+\bar{x})^{*}$. For $p \in K_{d}$, the $x$-saturation of $p$ is the element $\left|x^{\top}\right\rangle(p) \in K_{d}$.

ii) A covering set for $x$ is an element $q \in K_{d}$ such that $\left|x^{\top}\right\rangle(q) \geq 1$, i.e. whose $x$-saturation is total. A section of $x$ is a minimal covering set.

iii) A wide sub of $x$ is an element $w \leq x$ such that $|w\rangle=|x\rangle$ and $\langle w|=\langle x|$. A skeleton of $x$ is a minimal wide sub.

iv) Given a section $s_{0}$ of $x$, a strategy for $x$ relative to $s_{0}$ is a skeleton $\sigma$ of $x^{\top} s_{0}$ such that $s_{0} \sigma \leq s_{0}$.

Note that when $(\Phi, X)$ is a $(2,0)$-polygraph, we describe $\Phi$ in $K(\Phi, X)$ as the element $\phi:=\left\{1_{x} \mid x \in \Phi_{1}\right\} \cup\left\{1_{1_{a}} \mid a \in \Phi_{0}\right\}$. In $K(\Phi, X)_{1}$, which we recall is a Boolean MKA for 0-operations, the equivalence generated by $\phi$ corresponds to the 1-groupoid $\Phi^{\top}$, and a section corresponds to a choice of a representative 0 -cell 
for each connected component in $\Phi^{\top}$. A wide sub of $\phi$ is a subset $\psi$ such that for any 1-cell $x: a \rightarrow b \in \Phi_{1}$, there exists some parallel 1-cell $x^{\prime}: a \rightarrow b \in \Phi_{1}$ such that $1_{x^{\prime}} \in \psi$. A skeleton of $\phi$ therefore corresponds to the choice of a single 1-cell amongst the sets of parallel 1-cells in $\Phi$; it is thus not unique and does not coincide with $\phi$ in general. When $\Phi$ is convergent and $\left\{\sigma_{a}\right\}_{a \in \phi_{0}}$ is a strategy in the sense of Section 2, then $\sigma=\left\{1_{\sigma_{a}} \mid a \in \phi_{0}\right\}$ is a strategy for $\phi$ in $K(\phi, X)$ with respect to $\mathrm{nf}_{\phi}$. This result is proved for any convergent element of a MKA in Proposition 2.

By definition, a strategy $\sigma$ satisfies $d(\sigma)=d\left(x^{\top} s_{0}\right)=1$, and $r(\sigma)=$ $r\left(x^{\top} s_{0}\right)=s_{0}$. The following lemma states that a strategy contains the associated section:

Lemma 2. Given a section $s_{0}$ of $x$ and a strategy $\sigma$ for $x$ relative to $s_{0}$, we have $s_{0} \sigma=s_{0}$ and $s_{0} \leq \sigma$.

Proof. By hypothesis we have $s_{0} \sigma \leq s_{0}$. Showing that $s_{0} \sigma$ is a covering set allows us to deduce by minimality of $s_{0}$ that $s_{0} \leq s_{0} \sigma \leq \sigma$, which gives both desired conclusions. Since $\sigma$ is a strategy relative to $x$, we know that $\left\langle x^{\top} s_{0}\right|=\langle\sigma|$. We calculate the saturation of $s_{0} \sigma$

$$
\left\langle x^{\top}\right|\left(s_{0} \sigma\right)=r\left(s_{0} \sigma x^{\top}\right)=\left\langle x^{\top}\right|\langle\sigma|\left(s_{0}\right)=\left\langle x^{\top}\right|\left\langle x^{\top} s_{0}\right|\left(s_{0}\right) \geq\left\langle x^{\top}\right|\left(s_{0}\right) \geq 1,
$$

where we used properties of modalities for the first two steps, then the hypothesis that $\sigma$ is a strategy. To conclude, we used that $\left\langle x^{\top} s_{0}\right|\left(s_{0}\right) \geq\left\langle s_{0}\right|\left(s_{0}\right)=s_{0}$ and that $s_{0}$ is a covering set.

By conversion, we also get $\bar{\sigma} s_{0}=s_{0}$ and $s_{0} \leq \bar{\sigma}$. This immediately gives the following properties of a strategy $\sigma$ relative to a section $s_{0}$ :

$$
\sigma \cdot \sigma=\sigma, \quad \bar{\sigma} \cdot \bar{\sigma}=\bar{\sigma}, \quad \sigma \leq \sigma \cdot \bar{\sigma} \quad \text { and } \quad \bar{\sigma} \leq \sigma \cdot \bar{\sigma} .
$$

Indeed, $\sigma \sigma=\sigma s_{0} \sigma=\sigma s_{0}=\sigma$ by the fact that $r(\sigma)=s_{0}$ and Lemma 2, the case of $\bar{\sigma}$ follows by conversion. Additionally, $s_{0} \leq \bar{\sigma}$ so $\sigma=\sigma s_{0} \leq \sigma \bar{\sigma}$ and symmetrically for $\bar{\sigma}$.

Next, we will show that the normal forms and exhaustive iteration of a convergent element give us a section and a strategy, respectively. First, we show:

Lemma 3. Let $K$ a Boolean MKA. For a convergent element $x \in K$, we have $\left|x^{\top}\right\rangle=|\operatorname{exh}(x)\rangle\langle\operatorname{exh}(x)|$.

Proof. One direction holds since $\operatorname{exh}(x) \overline{\operatorname{exh}(x)} \leq x^{*} \bar{x}^{*} \leq x^{\top}$ so by monotonicity of taking diamonds and reversal of diamonds by conversion, we get $\left|x^{\top}\right\rangle \geq$ $|\operatorname{exh}(x)\rangle\langle\operatorname{exh}(x)|$. The other inequality is obtained via the star induction law for modalities (6). Indeed, it suffices to prove that

$$
|1\rangle+|x+\bar{x}\rangle|\operatorname{exh}(x) \overline{\operatorname{exh}(x)}\rangle \leq|\operatorname{exh}(x) \overline{\operatorname{exh}(x)}\rangle .
$$

We prove the inequality for each of the summands. We treat the case of $|1\rangle$ first: by definition,

$$
|\operatorname{exh}(x) \overline{\operatorname{exh}(x)}\rangle(p)=d\left(x^{*} \neg d(x) r\left(p x^{*}\right)\right)=d\left(x^{*} r\left(p x^{*}\right) \neg d(x)\right),
$$


where we used the so-called import-export law [5] $r(y p)=r(y) p$ for codomains and that multiplication is commutative in $K_{d}$. Since $p \leq 1$ we have

$$
p x^{*} r\left(p x^{*}\right) \neg d(x) \leq x^{*} r\left(p x^{*}\right) \neg d(x),
$$

and since $\left(p x^{*}\right) r\left(p x^{*}\right)=p x^{*}$, applying domain on both sides yields

$$
|\operatorname{exh}(x) \overline{\operatorname{exh}(x)}\rangle(p) \geq d\left(p x^{*} \neg d(x)\right)=p d(\operatorname{exh}(x))=p,
$$

where we used the import-export law for domains $d(p y)=p d(y)$ and Lemma 1. Thus $|\operatorname{exh}(x) \overline{\operatorname{exh}(x)}\rangle \geq|1\rangle$. The case of $|x\rangle$ follows by the star unfold axiom:

$$
|x\rangle\left|x^{*} \neg d(x) \bar{x}^{*}\right\rangle=\left|x x^{*} \neg d(x) \bar{x}^{*}\right\rangle \leq\left|x^{*} \neg d(x) \bar{x}^{*}\right\rangle .
$$

The final case follows by the hypothesis of confluence:

$$
\begin{aligned}
|\bar{x}\rangle\left|x^{*} \neg d(x) \bar{x}^{*}\right\rangle=\left\langle x|| x^{*}\right\rangle\langle\operatorname{exh}(x)| & \leq\left\langle x^{*}|| x^{*}\right\rangle\langle\operatorname{exh}(x)| \\
& \leq\left|x^{*}\right\rangle\left\langle x^{*}\right|\langle\operatorname{exh}(x)| \\
& \leq\left|x^{*}\right\rangle\left\langle\operatorname{exh}(x) x^{*}|=| x^{*} \neg d(x) \bar{x}^{*}\right\rangle,
\end{aligned}
$$

where we also used $\operatorname{exh}(x) x^{*}=\operatorname{exh}(x)$. Applying the star induction axiom for modalities, we obtain the result.

Now we are ready to relate exhaustion and normal forms to strategies and sections, respectively:

Proposition 2. If $x$ is convergent, then $n f_{x}$ is a section of $x$. Furthermore, any skeleton $\sigma$ of exh $(x)$ is a strategy for $x$ with respect to $n f_{x}$, and we have

$$
\sigma \leq n f_{x}+x^{+}, \quad \bar{\sigma} \leq n f_{x}+\bar{x}^{+} \quad \text { and } \quad \bar{\sigma} \sigma=n f_{x}
$$

Proof. First we show that $\mathrm{nf}_{x}$ is a section. It is a covering set since

$$
\left|x^{\top}\right\rangle\left(\mathrm{nf}_{x}\right) \geq|\operatorname{exh}(x)\rangle\left(\mathrm{nf}_{x}\right)=d(\operatorname{exh}(x))=1
$$

where the last step is by Lemma 1. Suppose now there is some $s \in K_{d}$ such that $s \leq \operatorname{nf}_{x}$ and $s$ is a covering set. Since $s \leq \mathrm{nf}_{x} \leq \neg d(x)$, the star unfold and antidomain axioms give $s \cdot \operatorname{exh}(x)=s$, so $\langle\operatorname{exh}(x)|(s)=s$.

Therefore $1=\left|x^{\top}\right\rangle(s)=|\operatorname{exh}(x)\rangle\langle\operatorname{exh}(x)|(s)=| \operatorname{exh}(x)\rangle(s)$, where we used Lemma 3. This means that

$$
s \geq\langle\operatorname{exh}(x)|| \operatorname{exh}(x)\rangle(s)=\langle\operatorname{exh}(x)|(1)=r(\operatorname{exh}(a))=\mathrm{nf}_{x},
$$

where the first inequality is by Lemma 1 , so we may conclude $\mathrm{nf}_{x}=s$, i.e. $\mathrm{nf}_{x}$ is minimal.

Now we show that a skeleton $\sigma$ of $\operatorname{exh}(x)$ is a strategy for $x$ relative to $\mathrm{nf}_{x}$. Note that $\left|x^{\top} \mathrm{nf}_{x}\right\rangle=\left|x^{\top}\right\rangle\left\langle\mathrm{nf}_{x}\right\rangle$ and $\left\langle x^{\top} \mathrm{nf}_{x}\right|=\left\langle\mathrm{nf}_{x}\right\rangle\left\langle x^{\top}\right|$. By Lemma 3,

$$
\left|x^{\top} \mathrm{nf}_{x}\right\rangle=|\operatorname{exh}(x)\rangle\left\langle\operatorname{exh}(x)\left|\left\langle\mathrm{nf}_{x}\right\rangle=\right| \operatorname{exh}(x)\right\rangle\left\langle\mathrm{nf}_{x}\right\rangle=|\operatorname{exh}(x)\rangle,
$$


since $\mathrm{nf}_{x} \operatorname{exh}(x)=\mathrm{nf}_{x}$, and $\operatorname{exh}(x) \mathrm{nf}_{x}=\operatorname{exh}(x)$. A symmetric proof gives $\left\langle x^{\top} \mathrm{nf}_{x}\right|=\langle\operatorname{exh}(x)|$. Since $\sigma$ is a skeleton of $\operatorname{exh}(x)$, its diamonds coincide with those of $\operatorname{exh}(x)$ and so, by what precedes, also with those of $x^{\top} \mathrm{nf}_{x}$. Since $\operatorname{exh}(x) \leq x^{\top} \mathrm{nf}_{x}, \sigma$ is a wide sub of $x^{\top} \mathrm{nf}_{x}$. Minimality of $\sigma$ as a wide sub follows from that same inequality plus the hypothesis that it is a skeleton of $\operatorname{exh}(x)$. To conclude, note that $\mathrm{nf}_{x} \sigma \leq \mathrm{nf}_{x} \operatorname{exh}(x)=\mathrm{nf}_{x}$. The first inequality follows from

$$
\sigma \leq \operatorname{exh}(x)=x^{*} \operatorname{nf}_{x}=\left(1+x x^{*}\right) \mathrm{nf}_{x} \leq \mathrm{nf}_{x}+x x^{*}=\mathrm{nf}_{x}+x^{+},
$$

where we used the definition of $\operatorname{exh}(x)$, the left star unfold axiom, $\mathrm{nf}_{x} \leq 1$ and the definition of the Kleene plus. The inequality for $\bar{\sigma}$ is then obtained by conversion. Finally, since $\sigma \leq \operatorname{exh}(x)$ and $x$ is confluent, we get

$$
\bar{\sigma} \sigma \leq \overline{\operatorname{exh}(x)} \operatorname{exh}(x)=\mathrm{nf}_{x} \bar{x}^{*} x^{*} \operatorname{nf}_{x} \leq \mathrm{nf}_{x} x^{*} \bar{x}^{*} \mathrm{nf}_{x}=\mathrm{nf}_{x},
$$

where we also used that $\mathrm{nf}_{x} \leq \neg d(x)=\neg r(\bar{x})$.

\section{Abstract coherence in 2-MKA}

Here we state and prove a formalisation of Theorem A in the context of globular modal 2-Kleene algebras. First we prove the main result of this paper:

Theorem 1 (Coherent normalising Newman's lemma). Let $K$ be a Boolean globular 2-Kleene algebra such that

i) $\left(K_{0},+, 0, \odot_{0}, 1_{0}, \neg_{0}\right)$ is a complete Boolean algebra,

ii) $K_{1}$ is continuous with respect to 0 -restriction, that is for all $\psi, \psi^{\prime} \in K_{1}$ and $\left(p_{\alpha}\right)_{\alpha} \subseteq K_{0}$ we have $\psi \odot_{0} \sup p_{\alpha} \odot_{0} \psi^{\prime}=\sup \left(\psi \odot_{0} p_{\alpha} \odot_{0} \psi^{\prime}\right)$.

Let $\phi \in K_{1}$ be convergent and $\sigma$ be a skeleton of exh $(\phi)$. If $A$ is a local confluence filler for $(\bar{\phi}, \phi)$, then $\left|\hat{A}^{*_{1}}\right\rangle_{1}\left(\sigma \odot_{0} \bar{\sigma}\right) \geq \bar{\phi}^{*_{0}} \odot_{0} \phi^{*_{0}}$.

Proof. We denote 0-multiplication by juxtaposition. First, we define a predicate $R N P$ expressing restricted normalised paving. Given $p \in K_{0}$, let

$$
R N P(p) \quad \Leftrightarrow \quad\left|\hat{A}^{*_{1}}\right\rangle_{1}(\sigma \bar{\sigma}) \geq \bar{\phi}^{*_{0}} p \phi^{*_{0}} .
$$

By completeness of $K_{0}$, we set $r:=\sup \{p \mid R N P(p)\}$ and by continuity of restriction we may infer $R N P(r)$. Furthermore, by downward closure of $R N P$, we have $R N P(p)$ if, and only if, $p \leq r$. We thereby deduce:

$$
\begin{aligned}
\forall p .\left(R N P\left(\left\langle\left.\phi\right|_{0} p\right) \Rightarrow R N P(p)\right)\right. & \Leftrightarrow \forall p .\left(\left\langle\left.\phi\right|_{0} p \leq r \Rightarrow p \leq r\right)\right. \\
& \left.\Leftrightarrow \forall p .(p \leq \mid \phi]_{0} r \Rightarrow p \leq r\right) \\
& \Leftrightarrow \mid \phi]_{0} r \leq r
\end{aligned}
$$

where we used the Galois connection (4). Thus, it suffices to show that

$$
\forall p .\left(R N P\left(\left\langle\left.\phi\right|_{0} p\right) \Rightarrow R N P(p)\right)\right.
$$


in order to conclude that $r=1_{0}$, by Noethericity of $\phi$. This method constitutes formalised Noetherian induction for Boolean MKA.

Given $p \in K_{0}$, we denote by $p_{\phi}$ the element $\left\langle\left.\phi\right|_{0}(p)=\mid \bar{\phi}\right\rangle_{0}(p)$. We have

$$
p \phi=p \phi r_{0}(p \phi)=p \phi\left\langle\left.\phi\right|_{0}(p) \leq \phi p_{\phi},\right.
$$

and similarly $\bar{\phi} p \leq p_{\phi} \bar{\phi}$. Using the star unfold axioms, we thereby deduce that

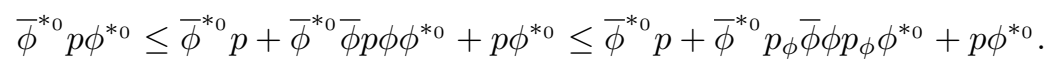

We first examine the middle summand:

$$
\begin{aligned}
\bar{\phi}^{* 0} p_{\phi} \bar{\phi} \phi p_{\phi} \phi^{*_{0}} \\
\quad \leq \bar{\phi}^{*_{0}} p_{\phi}|A\rangle_{1}\left(\phi^{*_{0}} \bar{\phi}^{*_{0}}\right) p_{\phi} \phi^{*_{0}} \\
\quad \leq\left|\bar{\phi}^{*_{0}} p_{\phi} A p_{\phi} \phi^{*_{0}}\right\rangle_{1}\left(\bar{\phi}^{*_{0}} p_{\phi} \phi^{*_{0}} \bar{\phi}^{*_{0}} p_{\phi} \phi^{*_{0}}\right) \\
\quad \leq|\hat{A}\rangle_{1}\left(\bar{\phi}^{*_{0}} p_{\phi} \phi^{*_{0}} \bar{\phi}^{*_{0}} p_{\phi} \phi^{*_{0}}\right) \\
\quad \leq|\hat{A}\rangle_{1}\left(\left|\hat{A}^{*_{1}}\right\rangle_{1}(\sigma \bar{\sigma}) \bar{\phi}^{*_{0}} p_{\phi} \phi^{*_{0}}\right) \\
\quad \leq|\hat{A}\rangle_{1}\left(\left|\hat{A}^{*_{1}}\right\rangle_{1}\left(\sigma \bar{\sigma} \bar{\phi}^{*_{0}} p_{\phi} \phi^{*_{0}}\right)\right) \\
\quad \leq|\hat{A}\rangle_{1}\left(\left|\hat{A}^{*_{1}}\right\rangle_{1}\left(\left|\hat{A}^{*_{1}}\right\rangle_{1}(\sigma \bar{\sigma} \sigma \bar{\sigma})\right)\right) \\
\quad \leq\left|\hat{A} \odot_{1} \hat{A}^{*_{1}} \odot_{1} \hat{A}^{*_{1}}\right\rangle_{1}(\sigma \bar{\sigma} \sigma \bar{\sigma}) \leq\left|\hat{A}^{*_{1}}\right\rangle_{1}(\sigma \bar{\sigma}) .
\end{aligned}
$$

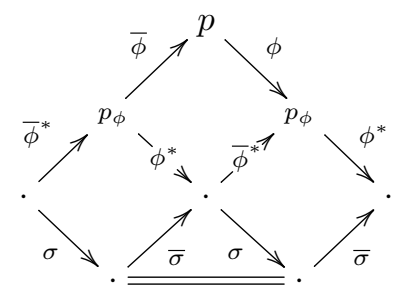

where we used that $A$ is a local confluence filler for the first step, then commutation of modalities with whiskering (9) and the definition of $\hat{A}(10)$ for the second and third steps. We then use the induction hypothesis $R P N\left(p_{\phi}\right)$ on the left instance of $\bar{\phi}^{* 0} p_{\phi} \phi^{* 0}$, followed by commutation of modalities with whiskering and whisker absorption (11), and then repeat for the instance on the right. Finally, we used that $\hat{A} \odot_{1} \hat{A}^{*_{1}} \leq \hat{A}^{*_{1}} \odot_{1} \hat{A}^{*_{1}} \leq \hat{A}^{*_{1}}$, monotonicity of taking diamonds and $\bar{\sigma} \sigma=\mathrm{nf}_{\phi}=r(\sigma)$, a consequence of Proposition 2 .

It remains to show that $\bar{\phi}^{* 0} p, p \phi^{*_{0}} \leq\left|\hat{A}^{*_{1}}\right\rangle_{1}(\sigma \bar{\sigma})$. First, observe that we have

$$
\begin{aligned}
\bar{\sigma} p \phi^{*_{0}} & =\bar{\sigma} p+\bar{\sigma} p \phi^{+_{0}} \\
& \leq \bar{\sigma}+\left(\mathrm{nf}_{\phi}+\bar{\phi}^{+0}\right) p \phi^{+_{0}} \\
& =\bar{\sigma}+\bar{\phi}^{+0} p \phi^{+0} \leq \sigma \bar{\sigma}+\bar{\phi}^{+0} p \phi^{+0} \leq\left|\hat{A}^{*_{1}}\right\rangle_{1}(\sigma \bar{\sigma}) .
\end{aligned}
$$

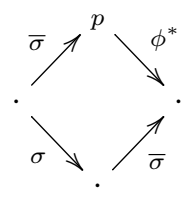

The first step is by the unfold axiom, the second uses Proposition 2 to bound $\bar{\sigma}$. The third step uses the fact that $\mathrm{nf}_{\phi}$ is a left annihilator for $\phi^{+0}$ since by definition we have $\mathrm{nf}_{\phi} \leq \neg d_{0}(\phi)$. Finally we use the fact that $\bar{\sigma} \leq \sigma \bar{\sigma}$ (12) coupled with $i d_{K_{1}}=\left|1_{1}\right\rangle_{1} \leq\left|\hat{A}^{*_{1}}\right\rangle_{1}$, i.e. reflexivity of $\hat{A}^{*_{1}}$, as well as the bound established by the previous calculation.

For convergent $\phi$, we have $d_{0}(\operatorname{exh}(\phi))=d_{0}\left(\phi^{*_{0}} \neg d_{0}(\phi)\right)=1_{0}$ by Lemma 1 . Since $\sigma$ is a skeleton of $\operatorname{exh}(\phi)$, we have $d_{0}(\sigma)=1_{0}$. By the converse axiom (2), this means that $\sigma \bar{\sigma} \geq 1_{0}$. Therefore, 


$$
\begin{aligned}
p \phi^{*_{0}} & \leq \sigma \bar{\sigma} p \phi^{*_{0}} \\
& \leq \sigma\left|\hat{A}^{*_{1}}\right\rangle_{1}(\sigma \bar{\sigma}) \\
& \leq\left|\hat{A}^{*_{1}}\right\rangle_{1}(\sigma \sigma \bar{\sigma})=\left|\hat{A}^{*_{1}}\right\rangle_{1}(\sigma \bar{\sigma}),
\end{aligned}
$$

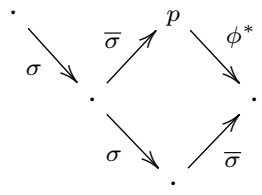

where we used commutation of whisker with modalities and whisker absorption, as well as $\sigma \sigma=\sigma(12)$. A symmetric argument yields $\bar{\phi}^{*_{0}} p \leq\left|\hat{A}^{*_{1}}\right\rangle_{1}(\sigma \bar{\sigma})$, concluding the proof.

The use of formalised Noetherian induction, as well as the calculation establishing the upper bound for the middle summand, are similar to those in the proof of Newman's lemma in [5]. Due to the fact that our result involves confluences in $\sigma$, the bounds for the outer summands require a different approach.

As a direct consequence of Theorem 1, we obtain the following result, which formalises Theorem A. Indeed, if $(\Phi, X)$ is a $(2,0)$-polygraph satisfying the corresponding hypotheses, Theorem 2 lifts the result to the power set when applied to $\phi:=\left\{1_{x} \mid x \in \Phi_{1}\right\} \cup\left\{1_{1_{a}} \mid a \in \Phi_{0}\right\}$ and $A=X$, viewed as elements of $K(\Phi, X)$. Following the argument given in Section 5, the conclusion asserts that for every zig-zag sequence $f: a \rightarrow b \in \Phi_{1}^{\top}$, there exists a 2-cell $\alpha_{f}: f \Rightarrow \sigma_{a} \star_{0} \sigma_{b}^{-}$obtained by whiskering and composing elements of $X$. In a 2-groupoid, this is equivalent to the existence of a 2 -cell $f \star_{0} \sigma_{b} \Rightarrow \sigma_{a}$.

Theorem 2 (Abstract coherence theorem). Let $K$ be a Boolean globular 2-Kleene algebra satisfying the additional hypotheses in Theorem 1 and $\phi \in K_{1}$ convergent. Given a normalisation strategy $\sigma$ and a local confluence filler $A$ for $(\bar{\phi}, \phi)$, we have

$$
\left|\hat{A}^{*_{1}}\right\rangle_{1}\left(\sigma \odot_{0} \bar{\sigma}\right) \geq \phi^{\top_{0}}=(\phi+\bar{\phi})^{*_{0}}
$$

Proof. We denote 0-multiplication by juxtaposition. As a result of Theorem 1 we have $\left|\hat{A}^{*_{1}}\right\rangle_{1}(\sigma \bar{\sigma}) \geq \bar{\phi}^{*_{0}} \phi^{*_{0}}$. By the star induction axiom, it suffices to show:

$$
1_{0}+(\phi+\bar{\phi})\left|\hat{A}^{*_{1}}\right\rangle_{1}(\sigma \bar{\sigma}) \leq\left|\hat{A}^{*_{1}}\right\rangle_{1}(\sigma \bar{\sigma}) .
$$

By (2) and Proposition 2, we have $\sigma \bar{\sigma} \geq d_{0}(\sigma)=1_{0}$, so by reflexivity of $\hat{A}^{* 1}$, i.e. $1_{1} \leq \hat{A}^{*_{1}}$, we have $1_{0} \leq\left|\hat{A}^{*_{1}}\right\rangle_{1}(\sigma \bar{\sigma})$. Furthermore, since $\phi \leq \bar{\phi}^{*_{0}} \phi^{*_{0}}$ we have:

$$
\phi\left|\hat{A}^{*_{1}}\right\rangle_{1}(\sigma \bar{\sigma}) \leq \bar{\phi}^{*_{0}} \phi^{*_{0}}\left|\hat{A}^{*_{1}}\right\rangle_{1}(\sigma \bar{\sigma}) \leq\left|\hat{A}^{*_{1}}\right\rangle_{1}(\sigma \bar{\sigma})\left|\hat{A}^{*_{1}}\right\rangle_{1}(\sigma \bar{\sigma}) \leq\left|\hat{A}^{*_{1}}\right\rangle_{1}(\sigma \bar{\sigma})
$$

The case of $\bar{\phi}$ is identical. We conclude via the star induction axiom.

\section{Outlook}

In this article, we have introduced a formalisation of the notion of strategy for convergent ARS and thereby obtained an abstract coherence theorem. This constitutes an initial result formalising cofibrant replacements of algebraic structures by rewriting, such as polygraphic resolutions from convergent SRS, [15]. 
In this perspective, the first step is to formalise the critical branching lemma, a coherent confluence result for SRS. Kleene algebra axioms only allow iteration on the left or right of expressions, but not in context. We expect a formalisation of coherent confluence for SRS using the structure of higher-dimensional quantales [26], similar to higher-dimensional semirings [3] but in which multiplication distributes over arbitrary sums. The second step consists in extending our formalisation of normalisation strategies to higher dimensions, necessary for constructing cofibrant replacements, for example polygraphic resolutions via convergent rewriting systems [15].

Another direction is found in the domain of concurrency theory. Concurrent Kleene algebras (CKA) [19] are a convenient extension of Kleene algebras. While similar to 2-MKAs, these are used to give semantics to concurrent languages and their corresponding proof systems. CKAs enrich classical Kleene algebras with an extra parallel composition operation alongside the classical sequential composition. In particular, CKAs have applications for validation of concurrent programs by formalising Hoare-like proof systems for parallel computations, similarly to MKAs which have applications to verification of hybrid systems [30] and program correctness [11]. We expect that our approach to abstract coherence proofs in 2-Kleene algebras can also find applications to formalisation of proof systems for verifying general concurrent systems, for example based on higherdimensional trace semantics of Higher-Dimensional Automata [25,9] (a form of higher-dimensional rewriting system), see e.g. $[7,8]$.

\section{References}

1. S. L. Bloom, Z. Ésik, and G. Stefanescu. Notes on equational theories of relations. Algebra Universalis, 33(1):98-126, mar 1995.

2. A. Burroni. Higher-dimensional word problems with applications to equational logic. Theoret. Comput. Sci., 115(1):43-62, 1993. 4th Summer Conference on Category Theory and Computer Science (Paris, 1991).

3. C. Calk, E. Goubault, P. Malbos, and G. Struth. Algebraic coherent confluence and higher-dimensional globular Kleene algebras, 2020. preprint, arXiv:2006.16129.

4. P.-L. Curien, A. Duric, and Y. Guiraud. Coherent presentations of a class of monoids admitting a garside family, 2021. arXiv 2107.00498.

5. J. Desharnais, B. Möller, and G. Struth. Termination in modal Kleene algebra. In Exploring new frontiers of theoretical informatics. IFIP 18th world computer congress, TC1 3rd international conference on theoretical computer science (TCS2004), pages 647-660. Boston, MA: Kluwer Academic Publishers, 2004.

6. J. Desharnais and G. Struth. Internal axioms for domain semirings. Sci. Comput. Programming, 76(3):181-203, 2011.

7. U. Fahrenberg, C. Johansen, G. Struth, and K. Ziemianski. Languages of higherdimensional automata. CoRR, abs/2103.07557, 2021.

8. U. Fahrenberg, C. Johansen, G. Struth, and K. Ziemianski. lr-multisemigroups and modal convolution algebras. CoRR, abs/2105.00188, 2021.

9. L. Fajstrup, E. Goubault, E. Haucourt, S. Mimram, and M. Raussen. Directed Algebraic Topology and Concurrency. Springer, 2016.

10. S. Gaussent, Y. Guiraud, and P. Malbos. Coherent presentations of Artin monoids. Compos. Math., 151(5):957-998, 2015. 
11. V. B. F. Gomes and G. Struth. Modal kleene algebra applied to program correctness. In J. S. Fitzgerald, C. L. Heitmeyer, S. Gnesi, and A. Philippou, editors, FM 2016: Formal Methods - 21st International Symposium 2016, Proceedings, volume 9995 of Lecture Notes in Computer Science, pages 310-325, 2016.

12. G. Gonthier, J. Lévy, and P. Melliès. An abstract standardisation theorem. In Proceedings of the Seventh Annual Symposium on Logic in Computer Science (LICS 1992), pages 72-81. IEEE Computer Society, 1992.

13. Y. Guiraud and P. Malbos. Higher-dimensional categories with finite derivation type. Theory Appl. Categ., 22:No. 18, 420-478, 2009.

14. Y. Guiraud and P. Malbos. Coherence in monoidal track categories. Math. Structures Comput. Sci., 22(6):931-969, 2012.

15. Y. Guiraud and P. Malbos. Higher-dimensional normalisation strategies for acyclicity. Adv. Math., 231(3-4):2294-2351, 2012.

16. Y. Guiraud and P. Malbos. Polygraphs of finite derivation type. Math. Structures Comput. Sci., 28(2):155-201, 2018.

17. N. Hage and P. Malbos. Knuth's coherent presentations of plactic monoids of type A. Algebr. Represent. Theory, 20(5):1259-1288, 2017.

18. T. Hoare, B. Möller, G. Struth, and I. Wehrman. Concurrent Kleene algebra and its foundations. J. Log. Algebr. Program., 80(6):266-296, 2011.

19. T. Hoare, B. Möller, G. Struth, and I. Wehrman. Concurrent Kleene algebra and its foundations. J. Log. Algebraic Methods Program., 80(6):266-296, 2011.

20. G. Huet. Initiation à la théorie des catégories. INRIA. Notes de cours du DEA "Fonctionalité, Structures de Calcul et Programmation" donné à l'Université Paris VII en 1983-84 et 1984-85, 1987.

21. B. Jónsson and A. Tarski. Boolean algebras with operators. I. Amer. J. Math., 73:891-939, 1951.

22. M. Lawson. Inverse Semigroups, The Theory Of Partial Symmetries. World Scientific Publishing Company, 1998.

23. S. Mac Lane. Natural associativity and commutativity. Rice Univ. Studies, 49(4):28-46, 1963.

24. P. Melliès. Axiomatic rewriting theory I: A diagrammatic standardization theorem. In A. Middeldorp, V. van Oostrom, F. van Raamsdonk, and R. C. de Vrijer, editors, Processes, Terms and Cycles: Steps on the Road to Infinity, volume 3838 of Lecture Notes in Computer Science, pages 554-638. Springer, 2005.

25. V. R. Pratt. Modeling concurrency with geometry. In D. S. Wise, editor, Conference Record of the Eighteenth Annual ACM Symposium on Principles of Programming Languages, 1991, pages 311-322. ACM Press, 1991.

26. K. I. Rosenthal. Quantales and their applications, volume 234 of Pitman Research Notes in Mathematics Series. Longman Scientific \& Technical, Harlow; copublished in the United States with John Wiley \& Sons, Inc., New York, 1990.

27. C. C. Squier, F. Otto, and Y. Kobayashi. A finiteness condition for rewriting systems. Theoret. Comput. Sci., 131(2):271-294, 1994.

28. R. Street. Limits indexed by category-valued 2-functors. J. Pure Appl. Algebra, 8(2):149-181, 1976.

29. G. Struth. Calculating Church-Rosser proofs in Kleene algebra. In Relational methods in computer science, volume 2561 of Lecture Notes in Comput. Sci., pages 276-290. Springer, Berlin, 2002.

30. J. J. H. y Munive and G. Struth. Verifying Hybrid Systems with Modal Kleene Algebra. In Relational and Algebraic Methods in Computer Science, pages 225-243. Springer International Publishing, 2018. 